\title{
Efeito do volume do tanque-rede na produtividade de tambaqui (Colossoma macropomum) durante a recria.
}

\author{
Levy de Carvalho GOMES ${ }^{1}$, Franmir Rodrigues BRANDÃO ${ }^{1,2}$, Edsandra Campos CHAGAS $^{1}$, Marcelo Frabizio \\ Barroncas FERREIRA ${ }^{3}$ e José Nestor de Paula LOURENÇO ${ }^{1}$
}

\begin{abstract}
RESUMO
O objetivo desta nota foi avaliar o efeito do volume do tanque-rede na produtividade de juvenis de tambaqui durante a recria. Juvenis de tambaqui foram criados por 60 dias, na densidade de 300 peixes $/ \mathrm{m}^{3}$, em tanques-rede de dois diferentes volumes: 1 e $6 \mathrm{~m}^{3}$. Foram avaliados parâmetros de crescimento e de produtividade final. Não houve diferença no crescimento em peso e em comprimento. O coeficiente de variação do comprimento foi significativamente menor para os peixes do tanque-rede pequeno. A sobrevivência, produção por volume e o ganho de peso não diferiram entre os tratamentos. Os peixes do tanque de maior volume foram significativamente mais eficientes na conversão alimentar. A recria pode ser realizada em tanques dos dois volumes testados sem prejuízo zootécnico para o criador.
\end{abstract}

PALAVRAS-CHAVE

Piscicultura, tanque-rede, produção, tambaqui, Colossoma.

\section{Effect of cage volume on productivity of tambaqui (Colossoma macropomum) during second growth phase.}

\begin{abstract}
The goal of this note was to evaluate the effect of cage volume on the productivity of juvenile tambaqui during second growth phase. Tambaqui juveniles were raised for 60 days on a density of $300 \mathrm{fish} / \mathrm{m}^{3}$, in two cages of different volumes: 1 and $6 \mathrm{~m}^{3}$. We evaluated growth and productivity parameters. There were no differences in fish weight and length. The coefficient of variation of length was significantly lower for fish in the small volume cage. There was no differences in survival, volume production, and weight gain between treatments. Fish from the larger volume cage were significantly more efficient in food conversion. The second growth phase may be undertaken in both cage volumes without losses to fish farmers.
\end{abstract}

\section{KEY WORDS}

Fish culture, cage, production, tambaqui, Colossoma.

A tecnologia de piscicultura em tanques-rede vem sendo amplamente difundida no Brasil, mostrando-se uma técnica promissora por conciliar o uso sustentável do meio ambiente com uma alta produtividade oriunda da utilização de altas taxas de estocagem (Beveridge, 1996; Chagas et al., 2003).

Os juvenis de espécies nativas normalmente disponíveis no mercado são de tamanho inferior ao tamanho mínimo utilizado para povoamento de tanque-rede de engorda $(10-12 \mathrm{~cm})$. Portanto, para que a criação de peixes em tanques-rede cresça no Brasil é necessário desenvolver um pacote de produção direcionado para recria, que é a fase de engorda de um juvenil de $2-5 \mathrm{~cm}$ até atingir $10-12 \mathrm{~cm}$.
Os tanques-rede mais utilizados em processos de produção são os de 1 e $6 \mathrm{~m}^{3}$. O tanque de $1 \mathrm{~m}^{3}$ é normalmente utilizado para a fase de recria e o de $6 \mathrm{~m}^{3}$ para a engorda, porém muitas vezes também é aproveitado para recria. O volume do tanque-rede deve estar em sintonia com o número necessário de peixes que o produtor deseja produzir, uma vez que os custos de obtenção e operacional do tanque-rede são proporcionais ao seu volume (Beveridge, 1996). Diante disto, este trabalho teve o objetivo de avaliar a produção de tambaqui em tanques-rede de dois volumes: 1 ( $1 \mathrm{~m}$ altura $\mathrm{x} 1 \mathrm{~m}$ comprimento $\mathrm{x} 1 \mathrm{~m}$ largura) e 6 $\left(1,5 \mathrm{~m}\right.$ altura $\mathrm{x} 2 \mathrm{~m}$ comprimento $\mathrm{x} 2 \mathrm{~m}$ largura) $\mathrm{m}^{3}$.

\footnotetext{
${ }^{1}$ Embrapa Amazônia Ocidental, CP 319, 69011-970, Manaus, AM. E-mail: levy@cpaa.embrapa.br.

${ }^{2}$ Centro Universitário Nilton Lins. Av. Prof. Nilton Lins 3259, CEP 69058-040, Manaus, AM.

${ }^{3}$ Instituto Nacional de Pesquisas da Amazônia - INPA/BADPI, CP 478, 69011-970, Manaus, AM.
} 
Juvenis de tambaqui $(2,47 \pm 0,05 \mathrm{~cm}$ e $0,24 \pm 0,01 \mathrm{~g}$; média \pm erro padrão) foram obtidos na fazenda Santo Antônio (Rio Preto da Eva, AM) e levados para 6 tanquesrede com malha de $20 \mathrm{~mm}$ entrenós, revestidos internamente por uma malha de multifilamento de $5 \mathrm{~mm}$ entrenós. Os tanques-rede foram instalados em um açude de 6 ha, localizado no Pesque-Pague San Diego (Manaus, AM). Foram testados tanques-rede de dois diferentes volumes ( 3 repetições para cada volume): 1 e $6 \mathrm{~m}^{3}$. Os peixes foram estocados na densidade de 300 juvenis $/ \mathrm{m}^{3} \mathrm{e}$ alimentados até a saciedade aparente três vezes ao dia, seis dias por semana, com ração comercial extrusada com $34 \%$ de $\mathrm{PB}$, durante 60 dias.

Nos dias 30 e 60 de criação foram capturados 30 peixes de cada tanque-rede, sendo estes anestesiados com benzocaína (100 mg/L; Gomes et al., 2001), pesados e medidos. Com o resultado da biometria foi possível calcular o crescimento em peso e comprimento, o coeficiente de variação do comprimento $[\mathrm{CV}=$ (desvio padrão do comprimento/comprimento médio) $\mathrm{x} 100]$ e a taxa de crescimento específico $($ TCE $=[(\ln$ peso tempo 1 - ln peso tempo 0)/tempo] x 100). Os parâmetros de produção final avaliados foram: sobrevivência (\%), produção por volume (peixes $\left./ \mathrm{m}^{3}\right)$, ganho de peso $(\mathrm{GP}=$ peso inicial peso final) e conversão alimentar aparente (CAA = consumo de ração/ganho de peso).

Foram avaliados seis vezes por semana o oxigênio dissolvido e a temperatura com um oxímetro digital e a cada 7 dias o $\mathrm{pH}$ com um potenciômetro digital, alcalinidade e dureza por titulação (Boyd, 1982) e a amônia total de acordo com Verdow (1978). A água para as análises foi coletada no interior dos tanques-rede a uma profundidade média de $50 \mathrm{~cm}$ e em um ponto externo distante 20 metros de onde os tanques estavam instalados.

Os resultados obtidos foram comparados entre tratamentos pelo teste t a 5\% de significância.

As variáveis de qualidade da água avaliadas não apresentaram diferença significativa entre os tanques de diferente volume. Os valores de qualidade da água obtidos no interior dos tanques foram muito semelhantes aos do açude. A água do açude apresentava as seguintes características físico-químicas (média \pm erro padrão): oxigênio $(\mathrm{mg} / \mathrm{L}) 7,3 \pm 0,8$; temperatura $\left({ }^{\circ} \mathrm{C}\right) 29,7 \pm 0,5 ; \mathrm{pH}$ (unidades) 6,7 $\pm 0,3$; dureza $(\mathrm{mg} / \mathrm{L}) 3,6 \pm 0,6$; alcalinidade (mg/L) 5,6 $\pm 1,9$ e amônia total (mg/L) 1,2 $\pm 0,4$. A qualidade da água do açude está dentro da faixa considerada ótima para criação de peixes por Boyd (1982).

De acordo com Jobling (1994) problemas de espaço afetam a taxa de crescimento, o que pode ser mais pronunciado em tanques-rede de pequeno volume. $\mathrm{O}$ crescimento em comprimento, peso e a TCE não apresentaram diferença significativa $(P>0,05)$ entre os volumes avaliados, em 30 e 60 dias (Tabela 1), significando que para este tempo de criação a disponibilidade de espaço não teve um efeito adverso sobre o crescimento dos peixes. Após 60 dias de criação, os peixes dos dois volumes de tanques-rede testados atingiram o tamanho médio final desejado para a fase de recria $(>10 \mathrm{~cm})$.
$\mathrm{O} \mathrm{CV}$ foi significativamente mais alto nos peixes do tanque-rede de maior volume, aos 60 dias de criação (Tabela 1). O tambaqui é um peixe gregário, com fortes relações sociais onde é normal o aparecimento de peixes dominantes, o que ocasiona grande heterogeneidade dos peixes produzidos (Araújo-Lima \& Goulding, 1997), sendo esta a principal explicação para o resultado obtido no tanque-rede de grande volume. No tanque-rede de pequeno volume, a dominância pode ter sido quebrada pelo pouco espaço disponível, como acontece para outras espécies como o pirarucu (Arapaima gigas) (Cavero et al., 2003). A dominância é quebrada pois o gasto energético para mantêla é muito alto (Jobling, 1994).

A CAA foi significativamente menor no tanque de maior volume (Tabela 2). No tanque de pequeno volume a área de alimentação é pequena fazendo com que a disputa por alimento seja maior, causando um gasto

Tabela 1 - Crescimento de juvenis de tambaqui durante a recria em tanques-rede de diferentes volumes. Os resultados são média \pm erro-padrão de três repetições de cada tratamento. * indica diferença significativa pelo teste $\mathrm{t}(\mathrm{P}<0,05)$; CV é o coeficiente de variação do comprimento e TCE a taxa de crescimento específico.

\begin{tabular}{lcc}
\hline & \multicolumn{2}{c}{ Dia de criação } \\
\cline { 2 - 3 } Volume do Tanque-rede $\left(\mathrm{m}^{3}\right)$ & 30 & 60 \\
\hline Comprimento $(\mathrm{cm})$ & & \\
1 & $5,88 \pm 0,26$ & $10,70 \pm 0,16$ \\
6 & $5,66 \pm 0,17$ & $10,54 \pm 0,28$ \\
\hline Peso (g) & & \\
1 & $3,89 \pm 0,35$ & $20,90 \pm 1,33$ \\
6 & $3,89 \pm 0,39$ & $21,53 \pm 1,69$ \\
\hline CV comprimento (\%) & & \\
1 & $17,94 \pm 1,20$ & $09,54 \pm 1,02$ \\
6 & $20,01 \pm 5,49$ & $14,71 \pm 0,90^{*}$ \\
\hline TCE $(\%)$ & & \\
1 & $9,25 \pm 0,33$ & $5,62 \pm 0,41$ \\
6 & $9,24 \pm 0,32$ & $5,70 \pm 0,44$ \\
\hline \hline
\end{tabular}

Tabela 2 - Produtividade de juvenis de tambaqui durante a recria em tanques-rede de diferentes volumes, após 60 dias de criação. Os resultados são média \pm erro-padrão de três repetições de cada tratamento. * indica diferença significativa pelo teste $\mathrm{t}$ $(\mathrm{P}<0,05)$.

\begin{tabular}{lcc}
\hline \hline & \multicolumn{2}{c}{ Volume do Tanque-rede $\left(\mathrm{m}^{3}\right)$} \\
\cline { 2 - 3 } Parâmetros & 1 & 6 \\
\hline Sobrevivência (\%) & $68,8 \pm 12,2$ & $64,9 \pm 11,09$ \\
Conversão alimentar & $1,27 \pm 0,17^{*}$ & $0,70 \pm 0,07$ \\
aparente & $203,3 \pm 36,7$ & $194,6 \pm 33,3$ \\
Produção (peixe $\left./ \mathrm{m}^{3}\right)$ & $3856 \pm 419$ \\
\hline Ganho de Peso $\left(\mathrm{g} / \mathrm{m}^{3}\right)$ & $4148 \pm 834$ & 34 \\
\hline
\end{tabular}




\section{ACTA \\ AMAZONICA}

EFEITO DO VOLUME DO TANQUE-REDE NA PRODUTIVIDADE

DE TAMBAQUI (Colossoma macropomum) DURANTE A RECRIA energético nos peixes que leva a um aumento no consumo de ração para manter as funções vitais e continuar crescendo. Os resultados de CAA obtidos neste estudo mostram que o juvenil de tambaqui foi mais eficiente em converter a ração do que outro Characiforme da Amazônia, o matrinxã (Gomes et al., 2000) durante a larvicultura em tanques.

A produção por volume e a sobrevivência foram semelhantes nos tanques de 1 e $6 \mathrm{~m}^{3}$ (Tabela 2), sendo este resultado inverso ao esperado, pois de acordo com Beveridge (1996) tanques de menor volume trocam de água mais rapidamente que tanques de maior volume, desta forma, apresentam uma maior produtividade. A sobrevivência média obtida neste estudo ( $\pm 65 \%)$ foi semelhante à obtida para o tambaqui nos sistemas de recria em viveiro (75\%) por Melo et al. (2001). A produção por volume na recria de tambaqui em tanque-rede ( \pm 200 peixes/ $\mathrm{m}^{3}$ ) foi muito mais alta que as obtidas para a recria em viveiros (Melo et al., 2001) e tanques (Souza et al., 1998) que foram respectivamente 7,5 e 20 peixes $/ \mathrm{m}^{2}$.

A produtividade de juvenis de tambaqui foi semelhante nos dois volumes de tanque. Desta forma, durante a recria, o criador pode escolher o tanque com volume adequado para suas necessidades sem preocupação com o desempenho zootécnico dos peixes criados.

\section{AGRADECIMENTOS}

Os autores agradecem aos senhores José P. de Souza e Asdrúbal Carreira pela ajuda prestada neste trabalho. Também agradecemos ao Senhor Hélcio Ferreira, proprietário do Pesque-Pague San Diego, por ter cedido o açude onde os tanques-rede foram instalados. Trabalho financiado pelo Projeto Tanque-rede/BASA e Projeto TANRE/FINEP.

\section{BIBLIOGRAFIA CITADA}

Araujo-Lima, C. R. M.; Goulding, M. 1997. So fruitful fish: ecology, conservation, and aquaculture of the Amazon's tambaqui. Columbia University Press, New York. 157pp.

Beveridge, M.C.M. 1996. Cage aquaculture. Fishing News Books, Oxford. 346pp.
Boyd, C.E. 1982. Water quality management for pond fish culture. Elsevier Science, Amsterdam. 317pp.

Cavero, B.A.S.; Pereira-Filho, M.; Roubach, R.; Ituassú, D.R.; Gandra, A.L.; Crescêncio, R. 2003. Efeito da densidade de estocagem na homogeneidade do crescimento de juvenis de pirarucu em ambiente confinado. Pesquisa Agropecuária Brasileira, 38:103-107.

Chagas, E.C.; Lourenço, J.N.P.; Gomes, L.C.; Val, A.L. 2003. Desempenho e estado de saúde de tambaquis cultivados em tanques-rede sob diferentes densidades de estocagem. In: Urbinati, E.C.; Cyrino, J.E.P. (Eds.). XII Simpósio Brasileiro de Aqüicultura. Aquabio, Jaboticabal, SP. p. 83-93.

Gomes, L.C.; Baldisserotto, B.; Senhorini, J.A. 2000. Effect of stocking density on water quality, survival, and growth of larvae of matrinxã, Brycon cephalus (Characidae), in ponds. Aquaculture, 183:73-81.

Gomes, L.C.; Chippari-Gomes, A.R.; Lopes, N.P.; Roubach, R.; Araujo-Lima, C.A.R.M. 2001. Efficacy of benzocaine as anesthetic for tambaqui juveniles (Colossoma macropomum). Journal of the World Aquaculture Society, 31:426-431.

Jobling, M. 1994. Fish bioenergetics. Chapman \& Hall, London. $294 \mathrm{pp}$.

Melo, L.A.S.; Izel, A.C.U.; Rodrigues, F.M. 2001. Criação de tambaqui (Colossoma macropomum) em viveiros de argila/ barragens no estado do Amazonas. Embrapa Amazônia Ocidental (Série Documentos 18), Manaus. 30pp.

Souza, R.A.L.; Melo, J.S.C.; Pereira, J.A.; Peret, A.C. 1998. Determinação da densidade de estocagem de alevinos de tambaqui Colossoma macropomum Cuvier, 1818 (Pisces; Characidae) no Estado do Pará - Brasil. Boletim Técnico do CEPTA, 11:39-48.

Verdow, H.; Vanechted, C.J.A.; Dekkers, E.M.J. 1978. Ammonia determination based on indophenol with sodium salicylate. Water Research, 12:399-402.

\author{
RECEBIDO EM 07/02/2003 \\ ACEITO EM 17/12/2003
}


